\title{
A Co-operative Numerical Analysis of Scotochromogenic Slowly Growing Mycobacteria
}

\author{
By L. G. WAYNE, ${ }^{1}$ T. M. DIETZ, ${ }^{2}$ C. GERNEZ-RIEUX, ${ }^{3}$ \\ P. A. JENKINS, ${ }^{4}$ W. KÄPPLER, ${ }^{5}$ G. P. KUBICA, ${ }^{6,10}$ \\ J. B. G. KWAPINSKI, ${ }^{7}$ GERTRUD MEISSNER, ${ }^{8}$ S. R. PATTYN, ${ }^{9}$ \\ E. H. RUNYON, ${ }^{2}$ K. H. SCHRÖDER, ${ }^{8}$ VELLA A. SILCOX, ${ }^{10}$ \\ A. TACQUET, ${ }^{3}$ M. TSUKAMURA, ${ }^{11}$ E. WOLINSKY ${ }^{12}$
}

\section{(Accepted for publication 5 February 197I)}

\section{SUMMARY}

A co-operative numerical taxonomic analysis of slowly growing scotochromogenic mycobacteria of Runyon's group II with no restrictions on numbers of characters contributed by individual participants, or on techniques employed to derive these characters, is reported. Of four scotochromogen clusters defined in this study, three were of named species (Mycobacterium flavescens, $M$. gordonae and $M$. scrofulaceum) and one, a small cluster of four strains, may represent a new species. Results from pooled data are compared to results obtained with data from individual participating laboratories. Immunologic data were treated separately and showed good correlation with the numerical analyses.

\section{INTRODUCTION}

Taxonomic analysis of the genus Mycobacterium is complicated by the disparities in growth and metabolic rates of members of different species, and by the necessity of using specialized tests, some of which are different from those used for study of other genera (Wayne, 1964; Wayne, 1967). Consequently, it is sometimes difficult to reconcile subgeneric definitions employed in different laboratories. To improve this situation, a number of investigators in this field have organized themselves into the International Working Group on Mycobacterial Taxonomy (IWGMT). Their intention is to develop efficient informal routes of communication and to undertake co-operative studies of selected sets of mycobacteria which can be examined jointly in a number of different laboratories. This is a report on one such study, involving I l laboratories.

The speciation of slowly growing, scotochromogenic mycobacteria has not yet been completely resolved to the satisfaction of most investigators (Tacquet et al. 1967; Wayne, Doubek \& Diaz, 1967; Tsukamura, 1969). Furthermore, there is no general agreement on

\footnotetext{
1 Veterans Administration Hospital, San Fernando, California, U.S.A.

2 Veterans Administration Hospital, Salt Lake City, Utah, U.S.A.

3 Institut Pasteur, Lille, France.

4 Institute of Preventive Medicine, Cardiff, South Wales.

5 Tuberkulose-Forschungsinstitut, Berlin, Germany (DDR).

6 Trudeau Institute, Saranac Lake, New York, U.S.A.

7 University of Manitoba, Winnipeg, Canada.

8 Tuberkulose Forschungsinstitut, Borstel über Bad Oldesloe, Germany.

${ }^{9}$ Institut voor Tropische Geneeskunde, Antwerp, Belgium.

10 National Communicable Disease Center, Atlanta, Georgia, U.S.A.

11 Chubu Chest Hospital, Obu Chita-Gun Aichi-Ken, Japan.

12 Metropolitan General Hospital, Cleveland, Ohio, U.S.A.
} 
the selection of tests upon which to base the speciation, nor on standardization of techniques for performing the tests. For these reasons, our co-operative study was based on a 'permissive' philosophy: instead of demanding that participants perform certain tests by precisely specified methods, each investigator was free to do whatever he chose with the cultures. All agreed to submit their raw results to a central co-ordinator, who would collate the diverse data for each organism in the set and perform numerical taxonomic analyses on the pooled information. This approach was hoped to provide not only new information on the taxonomy of the selected organisms, but also insight into the concept of using co-operative studies for establishing generally acceptable sets of tests for difficult genera. Both of these objectives are being met. As additional co-operative studies, at present under way, are completed, tests, and conditions for performing them, should be selected which are the most definitive for most or all of the species of mycobacteria.

\section{METHODS}

Sixty cultures, most of them fitting Runyon's group II (Wayne, Runyon \& Kubica, 1969), and a few representatives of other groups, included for comparative studies, were selected from the collection of the senior author and sent to the American Type Culture Collection (ATCC). Subcultures were prepared and lyophilized at ATCC, and samples of each were returned to the originating laboratory. After these preparations were confirmed as matching the characteristics of the cultures originally submitted, the ATCC distributed the collection, identified only by code numbers, to participants in the study. After completion of the study we learnt that some recipients of the cultures distributed as ATCC 19250 and 23422 had found them to be mixed cultures.

Initially, a total of 333 characters were coded for each culture. These characters were based on non-immunologic tests performed in eight of the co-operating laboratories (laboratories A to $\mathrm{H}$ ). All data were converted to simple binary (i.e. I or o) form for analysis, along lines described previously (Wayne, 1967). In addition to these 333 characters, immunologic, lipid pattern and pathogenic data were also submitted, and these were reserved for later correlation with the primary analysis. As pointed out by Jones \& Sneath (1970), it is useful to treat serologic data separately from numerical phenetic data; if the two systems are in agreement, the validity of the classification is supported.

It soon became apparent that some data could be deleted safely from the study, with improvement of results. Two types of data were deleted, as follows:

(I) 'Irrelevant' data were those which were identical for all organisms included in the study. Such data might be useful in a broader study of a greater variety of bacilli, but they obviously contributed no differentiation in the set under examination.

(2) 'Repetitious' data were generated when several investigators performed the same test, with the same distribution of results. In such cases, data from a single laboratory were selected, and the replications omitted. This prevented undue weighting of the more popular tests.

After deletion of irrelevant and repetitious data we were left with 140 characters, which were then employed in preparation of a conventional $n \times n$ matrix table of matches by highest single link, with negative matches included. Data from some tests were coded for quantitative differences, yielding more than one character per test (Sneath, I968; Wayne, 1967).

The complete data from each laboratory (i.e. no deletions of irrelevant or repetitious data) were also individually subjected to numerical analysis and the matrices plotted in the same sequence of cultures as evolved from the analysis of the pooled data. This permitted a 
comparison of the clustering effects achieved under each laboratory's scheme with the clustering obtained in the composite study.

Distribution of selected properties, including immunologic characteristics, among the clusters derived by numerical methods were also tabulated.

No attempt is made to present the full details of all the techniques employed at this time. Furthermore, no data are identified, in this paper, in terms of the laboratory from which they were derived. All participants agreed in advance that they would not withdraw their data from this publication, regardless of the results obtained or the interpretations agreed upon by the majority of participants.

\section{RESULTS}

The clustering behaviour of the cultures in this collection, as determined from the pooled data from all laboratories, gives seven clusters segregated at the 75 to $80 \%$ level of matching (Fig. I).

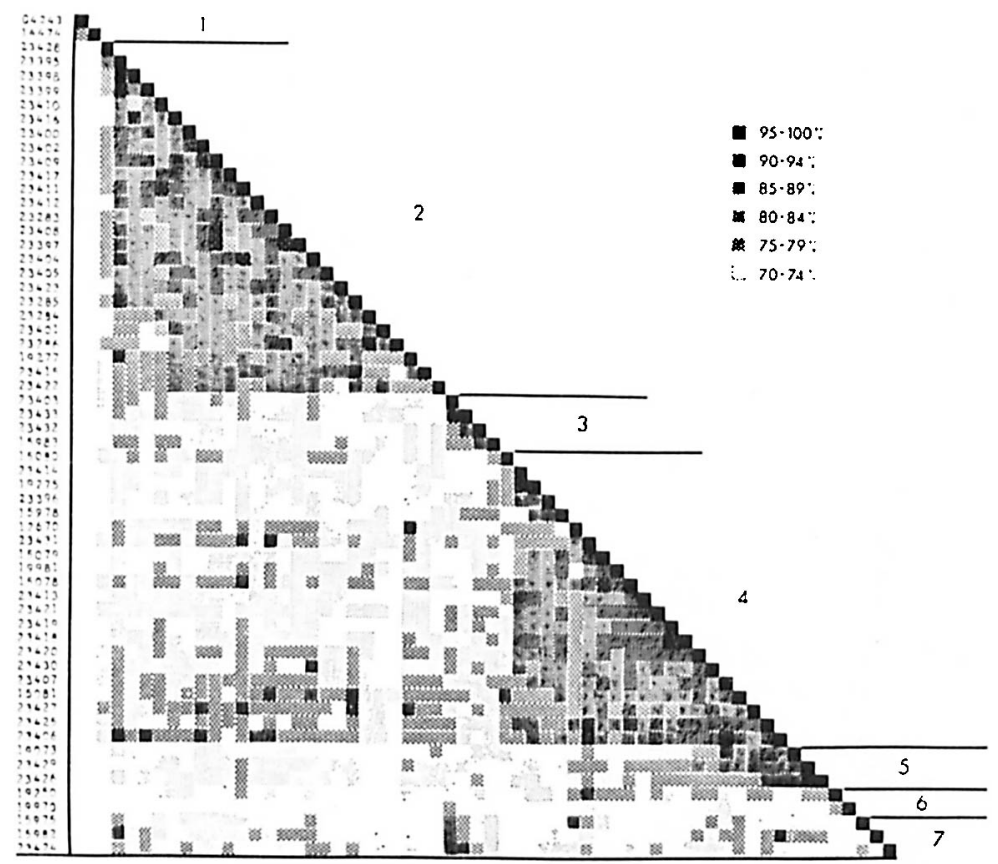

Fig. I. Matching matrix of 60 strains of mycobacteria, based on 140 pooled characters.

Cluster I consists of two strains at a $75 \%$ level of matching. One of these, ATCC 14474, is the type culture of Mycobacterium flavescens.

Cluster 2 is comprised of the so-called tap-water scotochromogens. Unfortunately, the type culture of Mycobacterium gordonae Bojalil, Cerbon \& Trujillo, 1962, was not included in this investigation, but the expanded description of this species (Pattyn, Hermans-Boveroulle \& van Ermengen, 1968; Tsukamura, 1970; Wayne, 1970) fits these organisms, and this cluster may be considered to represent strains of $M$. gordonae.

Cluster 3 is made up of four cultures intermediate between tap-water and scrofula scotochromogens, and may constitute a new species. No such designation is proposed at this time, though. 

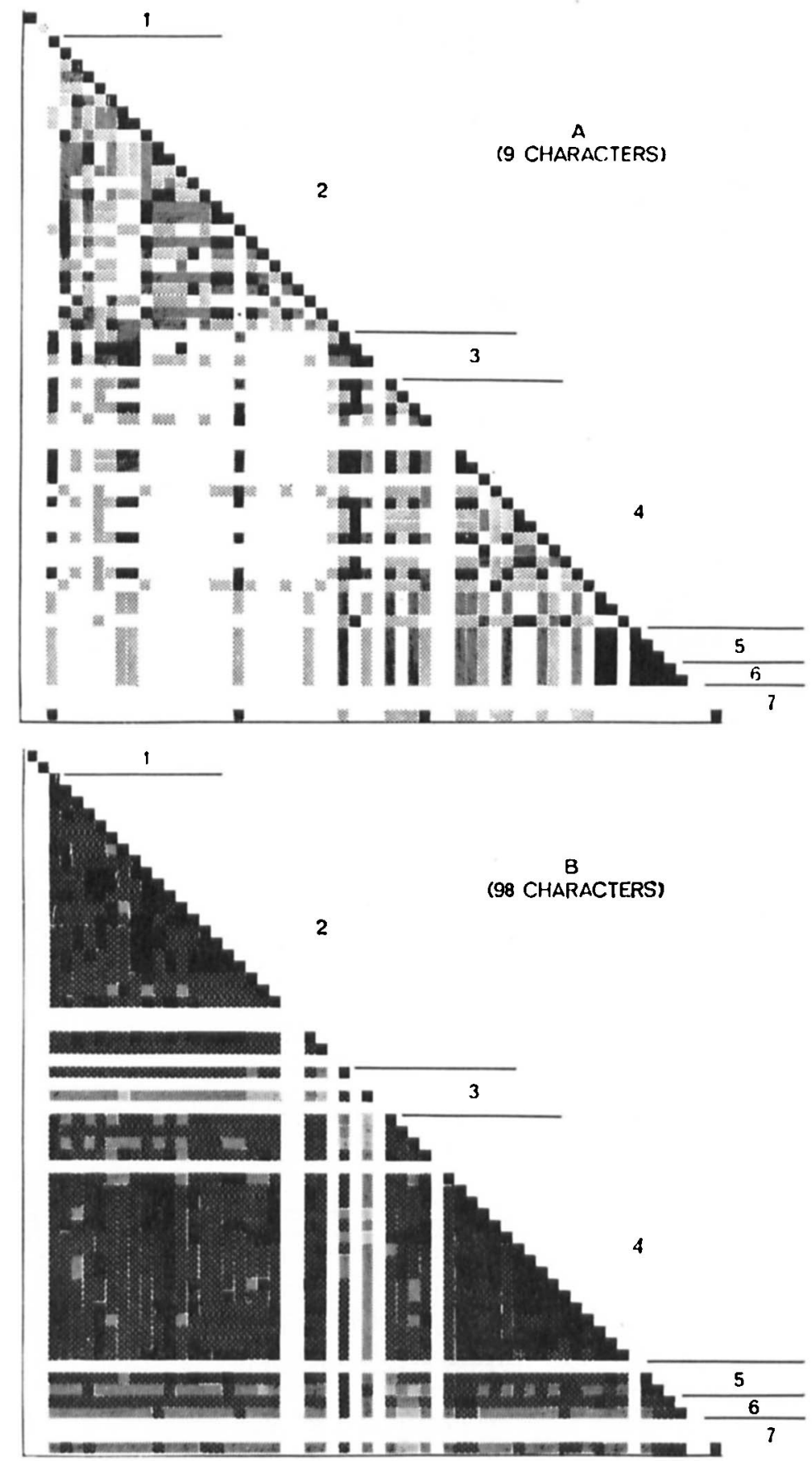

Fig. 2 A, B. Matching matrices of mycobacteria as determined from data from individual participating laboratories. The strains are presented in the same sequence as was employed for Fig. I. A black square along the diagonal represents identity of a strain with itself. For any culture that has no black square on the diagonal, that culture was not examined by the laboratory in question. Laboratories are identified only by code letters A to $\mathrm{H}$ (see also Fig. $2 \mathrm{C}$ to $\mathrm{H}$ ). Code for shading is the same as in Fig. I. 

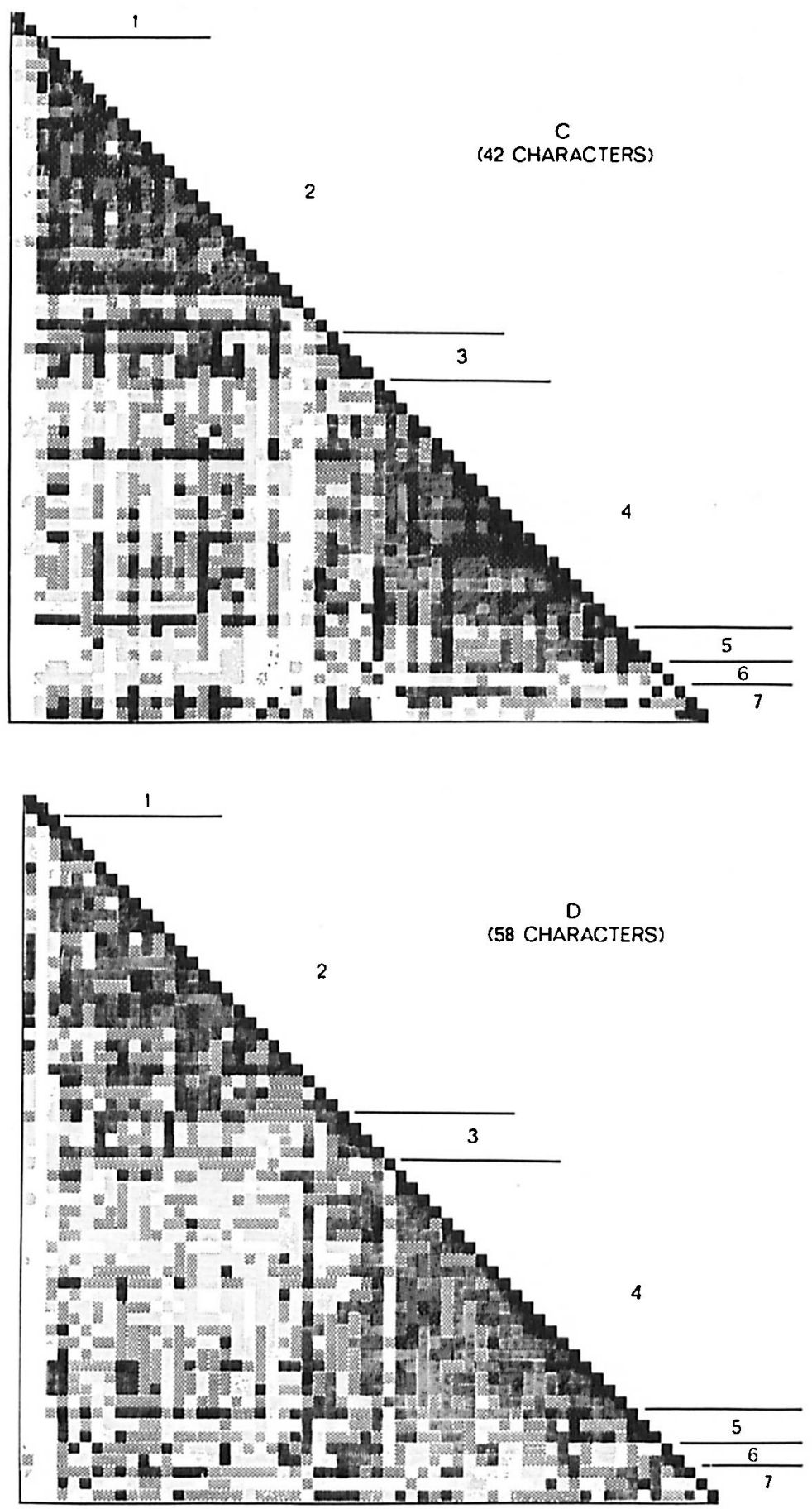

Fig. 2C, D. For legend see Fig. 2 A, B. 

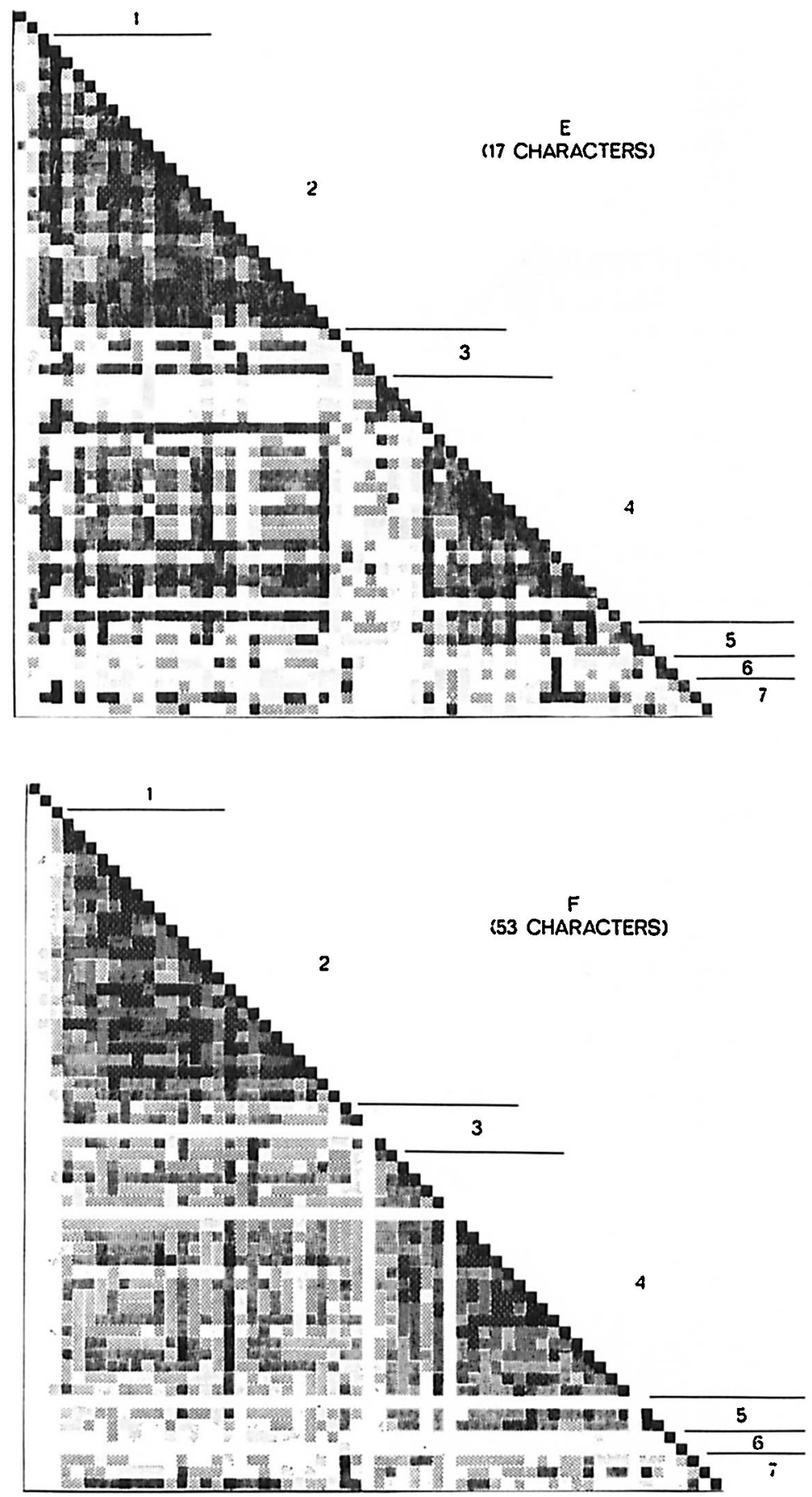

Fig. 2 E, F. For legend see Fig. 2 A, B. 

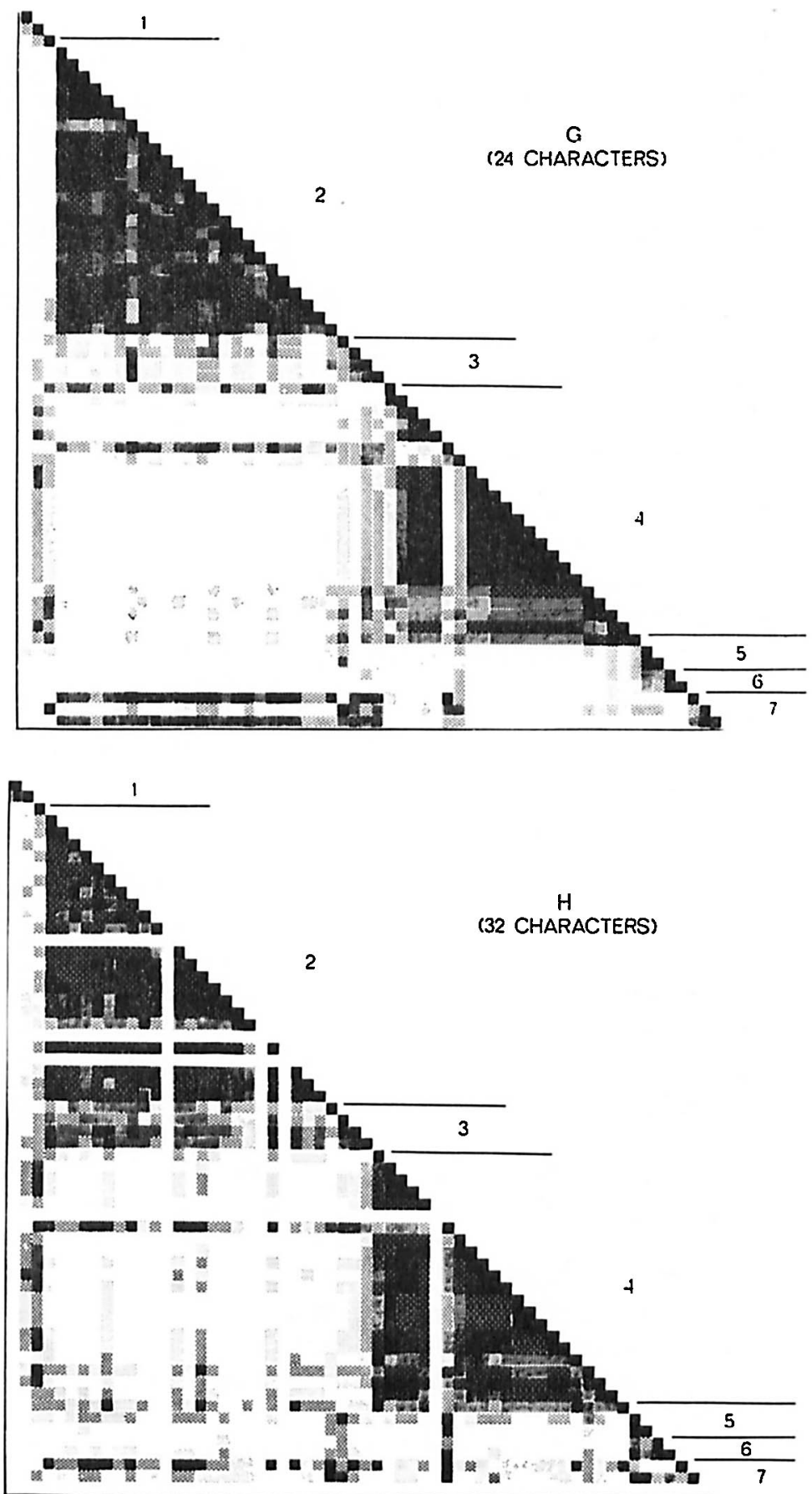

Fig. 2 G, H. For legend see Fig. 2 A, B. 
Cluster 4 includes ATCC I998I, the type culture of Mycobacterium scrofulaceum Prissick \& Masson, 1956, ATCC 19275, the catalogued representative strain of $M$. marianum Suzanne \& Penso (Penso, I953), ATCC 234I3 and 234I4, which were received from Dr G. Penso as rough and smooth strains, respectively, of $M$. marianum. Reasons for conservation of the name $M$. scrofulaceum for members of this cluster were given by Wayne \& Lessel (1969). The representative culture of $M$. paraffinicum Davis, Chase \& Raymond (I956), ATCC I2670, also falls into this group but the fit is a poor one and its status as a separate species is still open to question.

Cluster 5 consists of three strains. Two of these, ATCC 23426 and 23429 , had been included, for comparison purposes, as representatives of Mycobacterium intracellulare. The other, ATCC 19073, is listed in the ATCC catalogue as a strain of $M$. scrofulaceum and exhibits pigment, high catalase and urease activity, but overall it appeared markedly similar to $M$. intracellulare and also agglutinated as a Watson serotype. It may be an intermediate form.

Cluster 6 contains two cultures: ATCC I9250, the type culture of Mycobacterium xenopi Schwabacher (1959), and ATCC 19970, which also bears that name in the catalogue. (As indicated earlier, some recipients of ATCC 19250 found it to be mixed and its position must be viewed with reservations.)

Cluster 7 is a poorly related set of three cultures of unclear taxonomic position.

The numbers of characters derived from the tests employed in each of the different participating laboratories ranged from nine to 98 . Matching (M) matrices prepared separately from the total data which could be coded from each laboratory (Fig. 2) are in general agreement, in clustering behaviour, with the master matrix (Fig. I) in most cases. The median pair scores of strains within each of the two major clusters (Mycobacterium gordonae, cluster 2, and $M$. scrofulaceum, cluster 4) and median pair scores of members of cluster 2 compared to members of cluster 4 were analysed by plotting frequencies of $\% \mathbf{M}$ scores within those areas of the matrices corresponding to each of the two clusters, and in the area corresponding to the intercluster values, and determining the median values, as \% M (Table I). The number of characters represented by these scores was approximated from the total number of characters employed for each matrix by rounding to the nearest whole number. This, of course, does not necessarily mean that any two pairs within a cluster, which have the same $\% \mathrm{M}$ scores, differ from one another in terms of the same characters. With the exception of data from one laboratory, all median intra cluster scores exceeded $80 \%$, and with the exception of data from another laboratory all median intercluster scores were less than $80 \%$. Thus in laboratory $\mathrm{A}$, the median number of differences among strains within each of the two major clusters was two characters, with four characters median difference between pairs of strains from heterologous clusters. In laboratory $G$, the median intracluster difference was two characters, but nine characters appeared to separate members of heterologous clusters. In the master matrix of pooled data, a median intracluster difference of 26 and 25 characters was seen within $M$. gordonae and $M$. scrofulaceum respectively, and 39 characters separated the heterologous pairs.

Using some of the more definitive characteristics for each cluster, cluster I (Mycobacterium flavescens) evidently includes organisms that differ markedly from the more slowly growing scotochromogens, in the following respects. Mycobacterium flavescens grows at a rate between that of classical rapid and slow growers; reduces $\mathrm{NO}_{3}^{-}$vigorously; grows on $5 \% \mathrm{NaCl}$ medium; grows in the presence of $0.2 \%$ picric acid; produces opacity in Tween agar in I week and is inhibited by malachite green and methyl violet. These organisms can use three organic acids and seven sugars as sole carbon source, produce acid from seven sugars, and 
can use glucosamine and trimethylamine as sole sources of C and N. Mycobacterium flavescens has been reported to be closely related to $M$. acapulcensis (Pattyn et al. I968).

A series of 16 characters were found which permitted the most definitive differentiation between clusters 2 to 4 (Table 2). Twelve characters (no. I, 2, 3, 4, 5, 6, 7, 8, 9, I2, I 5, I6) differentiate most strains of Mycobacterium gordonae from $M$. scrofulaceum. Seven characters (no. 2, 7, 8, I0, I3, I4, I5) separate $M$. gordonae from the unnamed cluster 3 , and nine

Table I. Median matching of intracluster and intercluster pairs of strains falling into clusters 2 and 4

\begin{tabular}{|c|c|c|c|c|c|c|c|}
\hline \multirow[b]{3}{*}{ Laboratory } & \multirow[b]{3}{*}{$\begin{array}{l}\text { Total no. } \\
\text { characters }\end{array}$} & \multicolumn{6}{|c|}{ Median matching of pairs of strains } \\
\hline & & \multicolumn{2}{|c|}{ Within cluster 2} & \multicolumn{2}{|c|}{ Within cluster 4} & \multicolumn{2}{|c|}{$\begin{array}{c}\text { Between } \\
\text { clusters } 2 \text { and } 4\end{array}$} \\
\hline & & $\% \mathbf{M}^{*}$ & $\begin{array}{c}\text { No. } \\
\text { characters } \\
\text { difference }\end{array}$ & $\% \mathbf{M}$ & $\begin{array}{c}\text { No. } \\
\text { characters } \\
\text { difference }\end{array}$ & $\% \mathbf{M}$ & $\begin{array}{c}\text { No. } \\
\text { characters } \\
\text { difference }\end{array}$ \\
\hline Pool & 140 & 82 & 26 & 82 & 25 & 72 & 39 \\
\hline A & 9 & 78 & 2 & 76 & 2 & 60 & 4 \\
\hline B & 98 & 93 & 7 & 94 & 6 & 92 & 8 \\
\hline $\mathrm{C}$ & 42 & 86 & 6 & 86 & 6 & 74 & I I \\
\hline D & 58 & $8 \mathrm{r}$ & I I & 82 & IO & 75 & I5 \\
\hline $\mathbf{E}$ & I 7 & 87 & 2 & 80 & 3 & 79 & 4 \\
\hline$F$ & 53 & 86 & 7 & 85 & 8 & 78 & I2 \\
\hline G & 24 & 93 & 2 & 90 & 2 & 64 & 9 \\
\hline $\mathbf{H}$ & 32 & 92 & 3 & 93 & 2 & 67 & II \\
\hline
\end{tabular}

Table 2. Distribution of those characters which show greatest resolving power among clusters 2, 3 and 4

\begin{tabular}{|c|c|c|c|}
\hline \multirow[b]{3}{*}{ Cluster... } & \multicolumn{3}{|c|}{ Percentage of strains exhibiting character } \\
\hline & $\begin{array}{c}M . \\
\text { gordonae }\end{array}$ & $\begin{array}{c}\text { Mycobacterium } \\
\text { spp. }\end{array}$ & $\begin{array}{c}M . \\
\text { scrofulaceum }\end{array}$ \\
\hline & 2 & 3 & 4 \\
\hline \multicolumn{4}{|l|}{ Enzymic activity } \\
\hline I. Tween hydrolysis & 100 & 75 & 0 \\
\hline 2. Lipase & 84 & 25 & 19 \\
\hline 3. Phosphatase & 96 & 50 & 0 \\
\hline 4. $\alpha$-Esterase & 96 & 75 & $3 I$ \\
\hline 5. $\beta$-Esterase & 100 & 100 & 31 \\
\hline 6. Urease & 16 & 0 & 90 \\
\hline 7. Nicotinamidase & 4 & 100 & 86 \\
\hline 8. Pyrazinamidase & 4 & 100 & 86 \\
\hline \multicolumn{4}{|l|}{ Growth } \\
\hline 9. At $39^{\circ}$. & 8 & 25 & 90 \\
\hline Io. With nicotinamide as $\mathrm{N}$ source & 100 & 0 & 100 \\
\hline \multicolumn{4}{|l|}{ Resistant to } \\
\hline I I. Oleate, $0.025 \%$ & 64 & 0 & 95 \\
\hline 12. Isoniazid, Io $\mu \mathrm{g} . / \mathrm{ml}$. & 33 & 0 & 95 \\
\hline I3. 'I314 Th', 10 $\mu \mathrm{g} . / \mathrm{ml}$. & 88 & 0 & 65 \\
\hline I4. Cycloserine, Io $\mu \mathrm{g} . / \mathrm{ml}$. & 80 & 0 & 71 \\
\hline I5. Thiacetazone, I $\mu \mathrm{g} . / \mathrm{ml}$. & 96 & 25 & 29 \\
\hline${ }^{*}$ x 6. Ethambutol, $5 \mu \mathrm{g} . / \mathrm{ml}$. & 20 & 67 & 94 \\
\hline
\end{tabular}

* Ethambutol resistance data was submitted after completion of the numerical analysis and is not included in the similarity matrices. 
characters (no. I, 4, 5, 6, 9, I0, I I, I 2, I4) separate $M$. scrofulaceum from cluster 3. However, more strains similar to members of cluster 3 must be examined before a reliable definition of a new species may be attempted.

Similar data is not presented for differentiation of clusters 5 and 6 , as these are small and represent selected strains of species which are being examined in great detail in another co-operative study at present under way. Similarly, cluster 7 is too heterogeneous to permit such analysis.

As indicated earlier, immunologic and lipid pattern data were reserved for independent correlation with the results of the numerical phenetic analysis. Serotypes were established by performing agglutination tests with whole cell suspensions and appropriate sera. Nine of the serotypes detected in this study (see Table 3) have been described in terms of their distribution among the Runyon groups (Schaefer, 1965; Schaefer, I968). Thus serotypes Boone, III, IV, VI, Watson and Davis are almost exclusively associated with strains of Mycobacterium intracellulare, a species which meets the criteria for Runyon's group III, i.e. slow growers with very little or no pigmentation. Serotypes Lunning, Scrofulaceum and Gause, on the other hand, are mainly associated with group II organisms, i.e. slow growers with marked pigmentation even when grown in the dark.

The distribution of strains in terms of 13 recognized agglutinating serotypes, as determined by laboratory $\mathrm{E}$ (Table 3) makes it evident that the various serotypes occur almost exclusively within one or another cluster. There is an obvious need for extensive examination of the species Mycobacterial gordonae, as the sera presently available account for only five of the 24 strains examined. The appearance of two 'Boone' serotypes among $M$. gordonae strains was surprising, as this is generally considered to be an $M$. intracellulare serotype but as one of these two strains was received as a mixed culture it was not included in Table 3. Of three Lunning serotypes, two occurred in the small cluster 3 , indicating again that this cluster deserves further examination. Most of the $M$. scrofulaceum strains fell into unique serotypes. One strain, however, reacted with both 'III' and 'Davis' antiserum; these two serotypes are generally considered to be part of the $M$. intracellulare- $M$. avium complex.

Specificity of delayed hypersensitivity reactions was determined in laboratory I by intradermal injection of a set of partially purified mycobacterial culture filtrate antigens (sensitins) into guinea pigs which were sensitized by injection of the test organism (Magnusson, 1967) (Table 4). The sensitin which yielded the largest skin reaction was coded as positive for purposes of this analysis. If none of the reference sensitins elicited a reaction greater than $10 \mathrm{~mm}$. in diameter, the infecting strain was not considered to be characterized in this test. In general, results of sensitin testing correlated well with phenetic clustering behaviour. It is of interest that the strains which agglutinated as Lunning types failed to induce maximum hypersensitivity of Lunning sensitin, and five of the Mycobacterium scrofulaceum strains reacted maximally to Lunning sensitin.

These immunologic results are in accord with the generally accepted attitude that maximum hypersensitivity reactions are species related (Magnusson, 1967), whereas agglutinations are definitive at a subspecific level (Schaefer, 1965).

Cytoplasmic antigen serogrouping was performed in Laboratory $\mathbf{J}$ by an immunodiffusion technique employing the soluble portion of mycobacterial lysates against selected antisera (Kwapinski; Alcasid \& Pulser, 1970). Most of the test cultures were assigned to major serogroups numbers $\mathrm{I}$ to 6 , on the basis of the main cytoplasmic antigen factors detected by immunodiffusion (Table 5). Some strains were assigned to a seventh, non-homogeneous serogroup of scotochromogens, and others to an eighth group, described as a 'non-scotochromogen' group. Although there is some tendency of certain cytoplasmic antigen sero- 


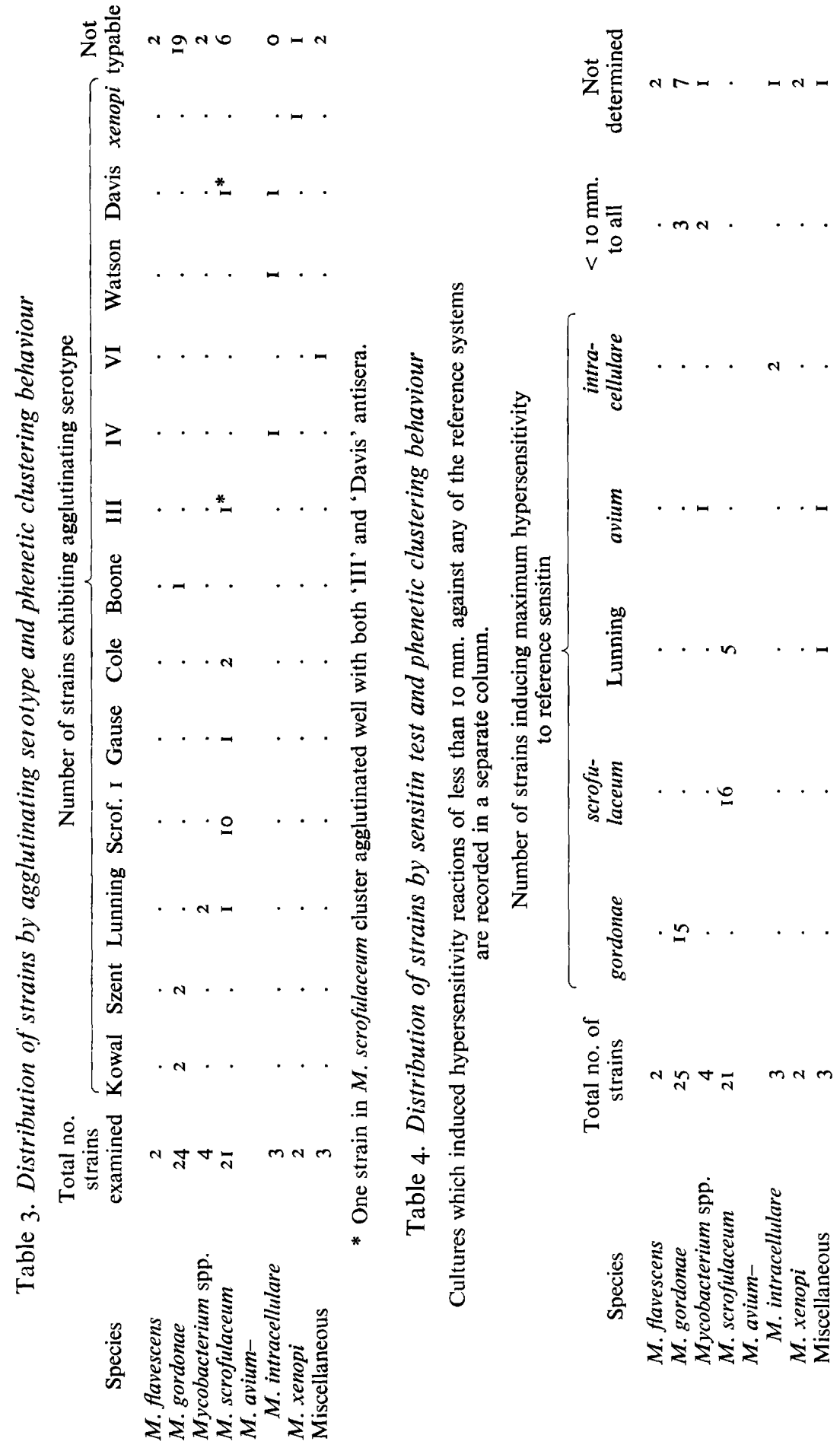

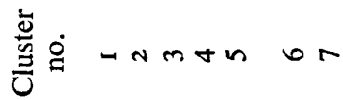


groups to dominate one or another of the clusters, the overlap observed indicated that these serogroups are not definitive for any of the species in question. There was no discernible pattern of relationship between the cytoplasmic antigen serogroups and the agglutinating serotypes described earlier.

Table 5. Distribution of strains by cytoplasmic antigen serogroup and phenetic clustering behaviour

\begin{tabular}{|c|c|c|c|c|c|c|c|c|c|c|}
\hline \multirow{2}{*}{$\begin{array}{c}\text { Cluster } \\
\text { no. }\end{array}$} & \multirow[b]{2}{*}{ Species } & \multirow{2}{*}{$\begin{array}{l}\text { Total no. } \\
\text { strains } \\
\text { examined }\end{array}$} & \multicolumn{8}{|c|}{$\begin{array}{c}\text { No. of strains falling into cytoplasmic } \\
\text { antigen serogroup no. }\end{array}$} \\
\hline & & & $\mathbf{I}$ & 2 & 3 & 4 & 5 & 6 & $7^{*}$ & $8 \dagger$ \\
\hline I & M. flavescens & 2 & I & I & . & . & . & . & $\cdot$ & $\cdot$ \\
\hline 2 & M. gordonae & 25 & I & 4 & 8 & 3 & 4 & I & 2 & 2 \\
\hline 3 & Mycobacterium spp. & 2 & I & . & . & $\mathrm{r}$ & . & $\cdot$ & . & . \\
\hline 4 & M. scrofulaceum & $2 \mathrm{I}$ & 5 & 3 & 2 & 4 & . & I & 3 & 3 \\
\hline 5 & $\begin{array}{l}\text { M. avium- } \\
\quad M . \text { intracellulare }\end{array}$ & 3 & $\mathbf{I}$ & I & . & I & $\cdot$ & $\cdot$ & $\cdot$ & $\cdot$ \\
\hline 6 & $M$. xenopi & 0 & . & . & . & . & . & . & . & . \\
\hline 7 & Miscellaneous & 3 & $\mathbf{I}$ & 2 & . & . & . & . & . & $\cdot$ \\
\hline
\end{tabular}

* Serogroup 7 consists of an assortment of scotochromogenic strains with patterns different from serogroups I to 6 .

$\dagger$ Serogroup 8 is described as 'non-scotochromogen' groups.

Table 6. Distribution of strains by lipid thin-layer chromatographic pattern and phenetic clustering behaviour

\begin{tabular}{|c|c|c|c|c|c|c|c|c|c|c|}
\hline \multirow{2}{*}{$\begin{array}{c}\text { Cluster } \\
\text { no. }\end{array}$} & \multirow[b]{2}{*}{ Species } & \multirow{2}{*}{$\begin{array}{l}\text { Total no. } \\
\text { strains } \\
\text { examined }\end{array}$} & \multicolumn{8}{|c|}{ No. of strains exhibiting lipid pattern } \\
\hline & & & A & B & $\mathrm{C}$ & $\mathbf{D}$ & $\mathrm{E}$ & $\mathrm{F}$ & G & $\mathbf{H}^{*}$ \\
\hline $\mathbf{I}$ & M. flavescens & 2 & . & . & & & . & I & . & $\mathbf{I}$ \\
\hline 2 & M. gordonae & 25 & . & . & 18 & 6 & . & . & $\mathbf{l}$ & . \\
\hline 3 & Mycobacterium spp. & 4 & . & . & 3 & . & . & . & . & I \\
\hline 4 & M. scrofulaceum & 20 & 8 & 5 & . & . & . & $\cdot$ & . & 7 \\
\hline 5 & M. avium- & & & & & & & & & \\
\hline & M. intracellulare & 3 & 2 & I & . & . & . & . & . & . \\
\hline 6 & M. xenopi & 2 & . & . & . & . & I & . & . & $\mathbf{I}$ \\
\hline 7 & Miscellaneous & 3 & I & . & I & . & . & . & . & I \\
\hline
\end{tabular}

By extracting lipids with ether-ethanol from the bacilli and subjecting the extracts to two-dimensional thin-layer chromatography (Marks \& Szulga, I965), laboratory K was able to assign the test strains to one of seven major groups (A to $G$ ) and to subdivisions within these groups on the basis of patterns of spots observed (Table 6). Thirteen of the 20 strains of Mycobacterium scrofulaceum (cluster 4) yielded patterns of major group A or B, and the remaining seven gave no distinctive pattern. The three strains of the $M$. avium-M. intracellulare complex also exhibited these lipid patterns. Separation into the groups A and B depended on the position of one or more yellow/orange spots in a small section of the chromatogram. These patterns were quite distinct from those of group $\mathrm{C}$ which were characterized by heavy brown/green spots and those of group D which were different again. However, all but one of the cultures of $M$. gordonae (cluster 2) yielded lipid patterns C or D, as did three of the strains in the unnamed cluster 3 . Subdivisions were made in the major groups where the overall patterns of the spots were similar, but there were small differences 


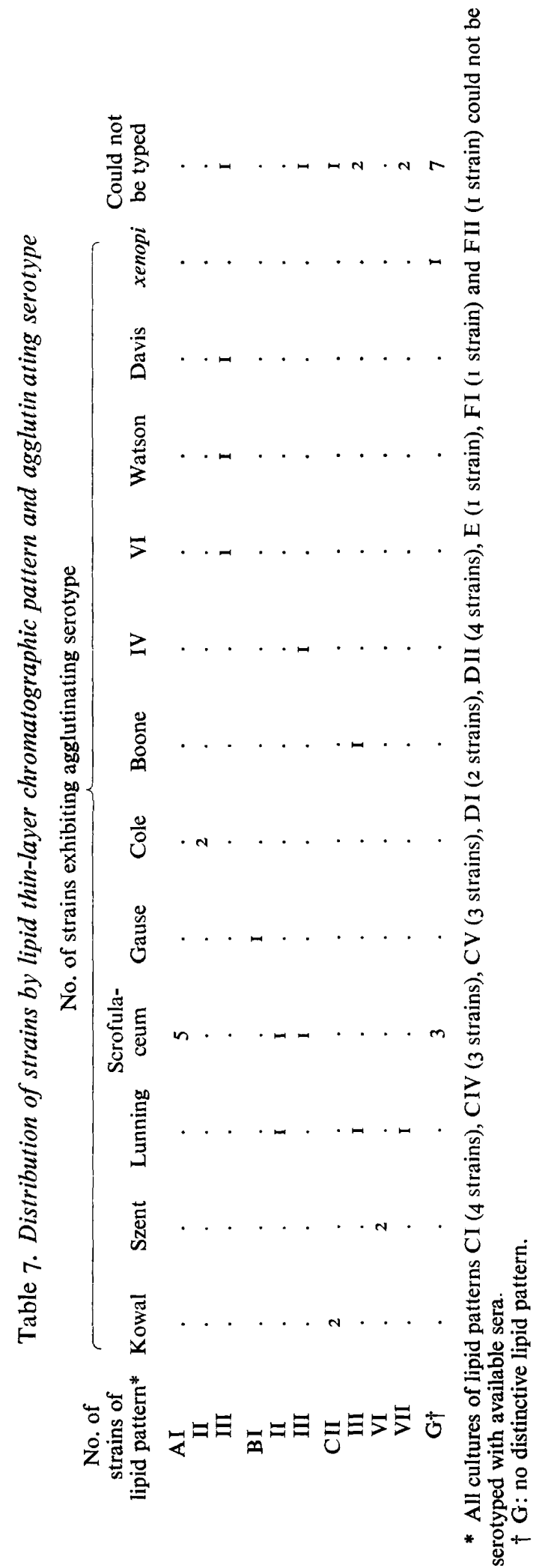


in the $R_{F}$ values or variations in the behaviour of the spots in different chromatography solvents. Analysis of these data in terms of the relationship between the lipid pattern and agglutinating serotype (Table 7) revealed that in some instances the subdivisions coincided with specific serotypes, e.g. the strains in groups AI, AII, CII, CVI, etc. The distribution of three of the four strains with an AIII lipid pattern into agglutinating serotypes associated with $M$. intracellulare suggests that AIII may represent strains unrelated to $M$. scrofulaceum which by phenetic and/or agglutination criteria comprise most strains of the major lipid patterns $\mathrm{A}$ and $\mathrm{B}$.

Table 8. Distribution of cultures in clusters 2, 3 and 4 in terms of source and clinical significance

For each entry, the denominator indicates the number of strains derived from the indicated source, and the numerator indicates the number of these cultures which were believed to be the cause of human disease.

\begin{tabular}{|c|c|c|c|}
\hline \multirow[b]{2}{*}{ Source } & \multicolumn{3}{|c|}{ Number of strains in cluster number } \\
\hline & 2 (M. gordonae) & $\begin{array}{c}3 \text { (Mycobacterium } \\
\text { spp.) }\end{array}$ & 4 (M. scrofulaceum) \\
\hline Soil, water, etc. & $0 / 7$ & . & $0 / 1$ \\
\hline Excised tonsils & $0 / 3$ & . & . \\
\hline Skin & $\cdot$ & . & $0 / 4$ \\
\hline Lesions of cervical adenitis & 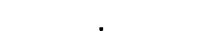 & . & $8 / 8$ \\
\hline Gastric lavage or sputum & I/I I & $2 / 3^{*}$ & $2 / 5^{*}$ \\
\hline Resected lung & $\therefore$ & $\mathbf{I} / \mathbf{I}$ & $2 / 2$ \\
\hline Not known & $? / 3$ & . & $\cdot$ \\
\hline Total no. clinically significant & $\mathbf{I}$ & 3 & 12 \\
\hline
\end{tabular}

Laboratory B investigated the ability of test strains to persist in spleens of mice after intravenous inoculation. The numbers of recoverable viable bacilli were tabulated on a 0 to 4 basis. The mean score and standard deviation for 22 strains of Mycobacterium gordonae was $I \cdot 9 \pm 0 \cdot 9$, and for I 8 strains of $M$. scrofulaceum it was $I \cdot 6 \pm I \cdot 2$. This technique offered no differentiation between these two species.

Because of the importance of mycobacteria in clinical microbiology, the distribution of strains in clusters 2 to 4 was correlated in terms of their sources and possible association with disease (Table 8). Only one of the 24 cultures of Mycobacterium gordonae had been considered significant. This agrees well with the observations of Kestle, Abbott \& Kubica (1967). However, this ratio may be disproportionately high for these tap-water organisms, for reasons discussed elsewhere (Wayne et al. 1967). Members of cluster 3, however, do possess the ability to cause human pulmonary disease. Members of the species $M$. scrofulaceum may be isolated under conditions where they are not causing disease, but they do have the ability to cause cervical lymphadenitis in children, and, infrequently, they also appear to cause pulmonary disease.

\section{DISCUSSION}

Some of the laboratories participating in this study are oriented primarily to identification of clinical isolates, whereas others are concerned with more general systematics within the genus Mycobacterium. As a consequence, the numerical taxonomic analysis of data from the laboratories with the former orientation may not accurately reflect their abilities to identify individual strains through weighting of data. Thus some of the participants recorded some 
observations on identity of the cultures either in terms of species names or of some special group codes. In most cases, organisms which were placed in a special group were found to fall in the same cluster when the pooled data were used for numerical analysis. Similarly, in most cases, the immunologic and lipid pattern analyses supported the numerical phenetic analysis.

A large number of characters should always be used in numerical taxonomic analyses and the selection of these characters should be 'unbiased'. When one deals with a genus which is markedly different from the genera most commonly encountered, as is the case with the genus Mycobacterium, it becomes evident that large numbers of inappropriate tests can introduce levels of 'noise' sufficient to obscure species differences.

Noise may be considered to be made up of several elements. Most obvious is simple experimental error. A second element is the inherent reliability of a given test and the variations introduced by factors in the test which are not well understood. A third element could be interphenon variation of strains. This does not represent error, but would reflect actual differences between strains. This, furthermore, could be considered noise, for general practical taxonomic purposes, only if the given variation did not correlate with other properties to produce a distinctive pattern (i.e. isolated interphenon variation). Thus a given phenon might be divided in half on the basis of any single character, but if continued addition of new characters to a scheme failed to support this division, it should be sufficient to describe this division without assigning new species status.

Noise, particularly of the second class described above, is an especially serious problem in a genus such as Mycobacterium whose members show such great variations in growth and metabolic rates (Wayne, 1964). The effectiveness of a set of tests may then be considered in terms of the difference between the number of characters serving to differentiate between pairs of strains from two different clusters and the number of characters showing difference between pairs of strains within the same cluster (noise). Thus the median noise level within cluster 2 does not differ by more than one character from the noise level in cluster 4 in any laboratory or in the pooled data (see Table I). In laboratories A, B and E, the difference between the median intercluster pair scores and the intracluster noise was two characters or fewer. This could be expected for the two laboratories which employed only nine and I7 characters respectively but was unexpected for the laboratory employing 98 characters. In contrast, laboratories $\mathrm{G}$ and $\mathrm{H}$, with only 24 and 32 characters, respectively, achieved resolution comparable to that seen with pooled data.

The large number of characters (98) from laboratory B did not, in fact, introduce a high noise level, as seen from the high intracluster $\% \mathrm{M}$ values; the intercluster score, however, differed very little from even this low noise level. These observations do not negate the value of these characters in consideration of the entire genus Mycobacterium. This study was limited to a narrow range of organisms and the data from laboratory B suggest close relationship between the two major species represented.

There was no intended implication, in selecting the range of cultures for this study, that species within Runyon's mycobacterial group II (which is defined only in terms of pigment and growth rate) bear any special relationship to one another but that they merely represented a convenient grouping of cultures which are commonly dealt with together. Furthermore, as only a few distinctions were previously recognized between Mycobacterium gordonae and $M$. scrofulaceum this presented a challenge to the concept of the permissive co-operative study. This concept is quite different from the usual approach to co-operative studies, in which a first goal is an evaluation of reproducibility of previously selected tests, as performed in different laboratories. This study began with the assumption that it was not 
known a priori which tests would prove to be productive and thus worthy of detailed scrutiny. As co-operative studies on members of the other Runyon groups are completed, the most definitive characters and techniques for determining these characters for speciation within the genus Mycobacterium will be identified. This will undoubtedly reinstate some characters which were deleted as irrelevant in the differentiation of species within the circumscribed group examined in this study. At that point a co-operative study on the reproducibility of the tests used in determining the selected characters should be established. The characters selected would be based on techniques employed in those laboratories which found the most consistent distribution of these characters within individual clusters. Ultimately, we hope to develop a secondary scheme of phenetic analysis for optimal resolution of all cultivable species within the genus Mycobacterium.

The authors are especially indebted to the following individuals who so capably dealt with the logistics of this investigation. Dr E. F. Lessel of the ATCC assumed responsibility for preparation and distribution of lyophilized cultures. Mrs Allie J. Broadway, of the Veterans Administration Hospital, San Fernando, California, transcribed all data for numerical analysis. Mr E. A. Warburton of the Veterans Administration Western Research Support Center, Sepulveda, California, prepared the programs and performed the computer analyses on which this report is based.

The authors are sorry for the poor quality of the figures but these had to be reproduced from copies of the originals, which were unfortunately destroyed in the February earthquake.

\section{REFERENCES}

Bojalil, L. F., Cerbon, J. \& Trujillo, A. (I962). Adansonian classification of mycobacteria. Journal of General Microbiology 28, 333-346.

Davis, J. B., ChASE, H. H. \& RAYMOND, R. L. (1956). Mycobacterium paraffinicum n.sp., a bacterium isolated from soil. Applied Microbiology 4, 310-315.

Jones, D. \& SNEATH, P. H. A. (1970). Genetic transfer and bacterial taxonomy. Bacteriological Reviews 34, $40-8 \mathrm{I}$.

Kestle, D. G., Aвbott, V. D. \& Kubica, G. P. (1967). Differential identification of mycobacteria. II. Subgroups of groups II and III (Runyon) with different clinical significance. American Review of Respiratory Disease 95, 1041-1052.

Kwapinski, J. B. G., AlCasid, A. \& Palser, H. (1970). Serologic relationships of endoplasm antigens of saprophytic mycobacteria. Canadian Journal of Microbiology 16, 871-876.

Magnusson, M. (1967). Identification of species of Mycobacterium on the basis of the specificity of the delayed type reaction in guinea pigs. Zeitschrift für Tuberkulose und Erkrankungen der Thoraxorgane 127, $55-56$.

MARKS, J. \& SzUlga, T. (1965). Thin layer chromatography of mycobacterial lipids as an aid to classification; technical procedures: Mycobacterium fortuitum. Tubercle, London 46, 400-4I I.

Pattyn, S. R., Hermans-Boveroulle, M. T. \& van Ermengen, J. (1968). A study on slow growing chromogenic (Runyon's group II) mycobacteria. Zentralblatt für Bakteriologie, Parasitenkunde, Infektionskrankheiten und Hygiene I Orig. 207, 509-5I6.

Penso, G. (1953). Criteri generali per determinare la posizione sistematica di un micobatterio. Atti del V symposium: Actinomycetales, morfologia, biologia e sistematica. International Congress for Microbiology 6, 89-101.

Prissick, F. H. \& MASSON, A. M. (1956). Cervical lymphadenitis in children caused by chromogenic mycobacteria. Canadian Medical Association Journal 75, 798-803.

SCHAEFER, W. B. (1965). Serologic identification and classification of the atypical mycobacteria by their agglutination. American Review of Respiratory Disease 92 Suppl., 85-93.

SCHAEFER, W. B. (1968). Incidence of the serotypes of Mycobacterium avium and atypical mycobacteria in human and animal diseases. American Review of Respiratory Diseases 97, 18-23. 
SChWABACher, H. (1959). A strain of Mycobacterium isolated from skin lesions of a cold-blooded animal, Xenopus laevus, and its relation to atypical acid-fast bacilli occurring in man. Journal of Hygiene $\mathbf{5 7}$, 57-67.

SNEATH, P. H. A. (1968). Vigour and pattern in taxonomy. Journal of General Microbiology 54, I-I I.

Tacquet, A., Tison, F., Plancot, M. Th., Devulder, B. \& Roos, Ph. (1967). Les mycobactéries scotochromogènes. Bulletin de l'Union internationale contre la tuberculose 39, 39-46.

TsUKAMURA, M. (1969). Identification of group II scotochromogens and group III non-photochromogens of mycobacteria. Tubercle, London 50, 5I-60.

TsukamuRA, M. (1970). Appropriate name for tap water scotochromogens. American Review of Respiratory Diseases 102, 643-644.

WAYNE, L. G. (1964). The mycobacterial mystique: deterrent to taxonomy. American Review of Respiratory Diseases 90, 255-256.

WAYNe, L. G. (1967). Selection of characters for an Adansonian analysis of mycobacterial taxonomy. Journal of Bacteriology 93, I382-139r.

WAYNE, L. G. (1970). On the identity of Mycobacterium gordonae Bojalil and the so-called tap water scotochromogens. International Journal of Systematic Bacteriology 20, I49-153.

WAYNE, L. G., DoubeK, J. R. \& DiAZ, G. A. (1967). Classification and identification of mycobacteria. IV. Some important scotochromogens. American Review of Respiratory Disease 96, 88-95.

WAYNE, L. G. \& LesSEL, E. F. (1969). On the synonymy of Mycobacterium marianum Penso 1952 and Mycobacterium scrofulaceum Prissick \& Masson 1956 and the resolution of a nomenclatural problem. International Journal of Systematic Bacteriology 19, 257-26r.

WAYNE, L. G., RunYon, E. H. \& KuBICA, G. P. (1969). Mycobacteria: a guide to nomenclatural usage American Review of Respiratory Diseases 100, 732-734. 